\title{
Response of microbial activity to dynamic flow conditions in the vadose zone: A modeling study \\ SWAMINI KHURANA ${ }^{1}$, FALK HESSE ${ }^{2}$ AND MARTIN THULLNER $^{2}$ \\ ${ }^{1}$ Helmholtz Centre for Environmental Research - UFZ, Leipzig \\ ${ }^{2}$ Helmholtz Centre for Environmental Research - UFZ \\ Presenting Author: swamini.khurana@ufz.de
}

The Critical Zone is a temporally dynamic natural system. Temporal dynamics in surficial processes such as weather events lead to mobilization and transport of organic matter from the surface to deeper into the subsurface. The resulting input of freshly derived dissolved organic matter (DOM) influences the microbial activity and diversity in the shallow subsurface. Additionally, the inherent spatial heterogeneity of the subsurface also impacts microbial activity. As a consequence, the dynamics of biogeochemical cycles is highly variable in space and time and governed by the temporal dynamics of processes at the surface. The vadose zone exhibiting also a variable and dynamic water saturation is the connection between surficial dynamics and the saturated aquifer. It needs to be studied for its ability to buffer the effects of surficial processes on this deeper subsurface compartments.

In this study, we undertake a numerical modeling approach to study the impact of spatio-temporal heterogeneities on nutrient cycles in the vadose zone. We used an already established biogeochemical process network, and an established numerical tool [1] to simulate microbially driven transformations of different organic carbon and nitrogen compounds in spatially heterogeneous domains and in different temporal dynamics scenarios, driven by data obtained from a subject site (AquaDiva Critical Zone Observatory, Hainich National Park, Thuringia, Germany [2]).

The obtained results support characterizing microbial activity in space and time with respect to their link to surficial processes, their impact on nutrient discharge to aquifers, and the memory of the system. Furthermore, the results provide the basis for the consideration of heterogeneity effects in effective rate descriptions of biogeochemical processes.

Refs:

[1] Centler et al., 2010. Computers \& Geosci. 36: 397-405.

[2] Küsel et al., 2016. Frontiers in Earth Science, 4: 32. 